\title{
Thermal Optical Properties of Lunar Dust Simulants and Their Constituents
}

\author{
James R. Gaier, ${ }^{1}$ Shaneise Ellis, ${ }^{2}$ and Nichole Hanks ${ }^{3}$ \\ NASA Glenn Research Center, Cleveland, OH 44135
}

\begin{abstract}
The total reflectance spectra of lunar simulant dusts $(<20 \mu \mathrm{m}$ particles $)$ were measured in order to determine their integrated solar absorptance $(\alpha)$ and their thermal emittance $(\varepsilon)$ for the purpose of analyzing the effect of dust on the performance of thermal control surfaces. All of the simulants except one had a wavelength-dependant reflectivity $(\rho(\lambda))$ near 0.10 over the wavelength range of 8 to $25 \mu \mathrm{m}$, and so are highly emitting at room temperature and lower. The $300 \mathrm{~K}$ emittance $(\varepsilon)$ of all the lunar simulants except one ranged from 0.78 to 0.92. The exception was Minnesota Lunar Simulant 1 (MLS-1), which has little or no glassy component. In all cases the $\varepsilon$ was lower for the $<20 \mu \mathrm{m}$ particles than for larger particles reported earlier. There was considerably more variation in the lunar simulant reflectance in the solar spectral range $(250$ to $2500 \mathrm{~nm})$ than in the thermal infrared. As expected, the lunar highlands simulants were more reflective in this wavelength range than the lunar mare simulants. The integrated solar absorptance $(\alpha)$ of the simulants ranged from 0.39 to 0.75 . This is lower than values reported earlier for larger particles of the same simulants (0.41 to 0.82$)$, and for representative mare and highlands lunar soils (0.74 to 0.91$)$. Since the $\alpha$ of some mare simulants more closely matched that of highlands lunar soils, it is recommended that $\alpha$ and $\varepsilon$ values be the criteria for choosing a simulant for assessing the effects of dust on thermal control surfaces, rather than whether a simulant has been formulated as a highlands or a mare simulant.
\end{abstract}

\section{Introduction}

A S NASA prepares to return humans to the Moon, extensive efforts are under way to design the thermal control systems that will protect future lunar landers and surface system elements from the harsh thermal environment. Part of this effort is to determine the effects of dust deposited on thermal control surfaces. But even a casual glance at the Moon reveals that the lunar surface is not uniform in brightness. The albedo of specific areas of the Moon varies from 0.50 for the brightest features to 0.07 for the darkest. ${ }^{1}$ If the dust is darker, then more light will be absorbed by it, resulting in increased heating both of the dust and, through conduction and radiation, any thermal control surface it may land on. To quantify these effects, lunar simulants must be developed that have a variety of reflectance properties.

There are several characteristics of the albedo which limit its usefulness when assessing the effects of radiant energy on thermal control systems. First, as these measurements are typically made either from Earth-based instruments or from lunar orbit, the topographical features within the measurement frame will affect the measurement. Any shadowed areas will skew the albedo to a lower value. And even for those areas lit, the fraction of light reflected is dependent on the angle of incidence. The bidirectional reflectance function of the lunar surfaces is known to be decidedly non-Lambertian. In addition, the albedo is typically measured in the visible region of the spectrum (400 to $700 \mathrm{~nm}$ wavelength), and the solar radiation is principally emitted over a wider spectral region, 250 to $2500 \mathrm{~nm}$ (Fig. 1). ${ }^{2}$

\footnotetext{
${ }^{1}$ Research Physicist, Space Environment and Experiments Branch, 21000 Brookpark Road, and Member AIAA.

${ }^{2}$ Summer Intern, UNCFSP/NASA Science and Technology Institute for Minority Institutions, 21000 Brookpark Road.

${ }^{3}$ Summer Intern, Lewis Educational Research Collaborative Internship Program, 21000 Brookpark Road.
} 


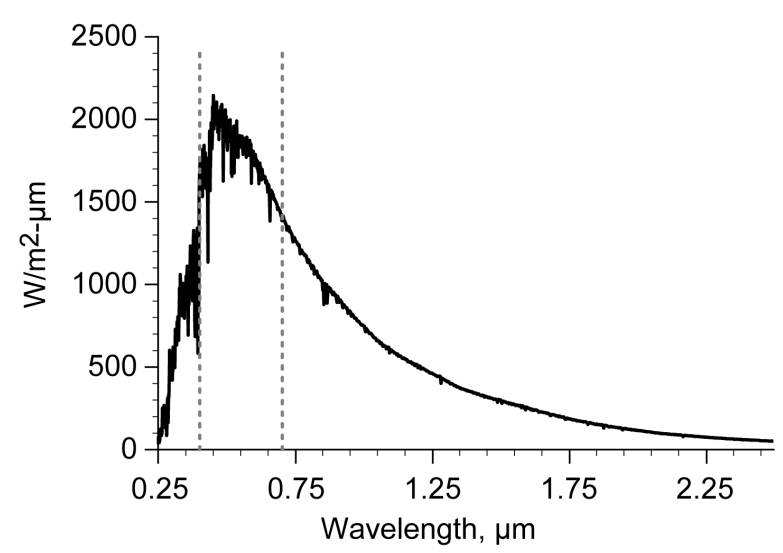

Figure 1. The solar spectrum measured at $1 \mathrm{AU}$ from the Sun above the Earth's atmosphere (Air Mass Zero spectrum). The visible spectrum is the region between the dotted lines.

More useful measures for most analyses are intrinsic material properties such as the wavelength dependant absorptivity $(\alpha(\lambda))$, reflectivity $(\rho(\lambda))$ and transmissivity $(\mathrm{T}(\lambda)$ ). These three properties are related through conservation of energy, since a photon must either be absorbed, reflected, or transmitted as described by Eq. (1):

$$
\alpha(\lambda)+\rho(\lambda)+\mathrm{T}(\lambda)=1
$$

Under the usual condition for the lunar surface where the surface is opaque $(T=0)$, Eq. (1) can be expressed as:

$$
\alpha(\lambda)=1-\rho(\lambda)
$$

Another useful relationship is Kirchhoff's Law, which states that for a given wavelength of light the emissivity $(\varepsilon(\lambda))$ of a surface is equal to its $\alpha(\lambda)$, or:

$$
\varepsilon(\lambda)=\alpha(\lambda)
$$

In thermal analysis the quantity usually required is the total integrated absorptance $(\alpha)$ and total integrated emittance (E). As illustrate in Fig. 1, convolving the $\alpha(\lambda)$ of a material over the 250 to $2500 \mathrm{~nm}$ range with the AM0 solar spectrum yields a very good approximation of $\alpha$, since there is little solar intensity outside this spectral range.

The intensity of $\varepsilon(\lambda)$ is temperature dependant. Temperatures of interest range from the hottest surfaces of a nuclear reactor (perhaps $800 \mathrm{~K}$ ) to the coldest of the permanently shadowed craters (perhaps $25 \mathrm{~K}$ ), though the daily lunar temperature swing of 100 to $400 \mathrm{~K}$ will encompass the temperatures most thermal control surfaces will be exposed to. The relationship between wavelength and temperature can be approximated by the Raleigh-Jeans law, which determines the wavelength at which the intensity for a blackbody radiation curve at a given temperature is maximum. Temperatures of interest and their corresponding wavelengths are shown in Table 1.

Table 1. Temperatures of interest and their corresponding wavelengths according to the Raleigh-Jeans law

\begin{tabular}{lcc}
\hline \multicolumn{1}{c}{ Feature } & Temperature, & Wavelength max, \\
& $\mathbf{K}$ & $\boldsymbol{\mu} \mathbf{m}$ \\
\hline Permanently shadowed craters & 25 & 115 \\
Lunar night & 100 & 29 \\
Lunar polar regions & 200 & 14 \\
Lunar habitat temperature & 300 & 10 \\
Lunar equator at noon & 400 & 7 \\
Nuclear reactor radiator & 800 & 4 \\
\hline \hline
\end{tabular}

Lunar simulant development is a rapidly evolving field. Although thermal optical properties of the lunar regolith are critical to designing systems that will survive the challenging lunar thermal environment, there has been little 
work to characterize the thermal optical properties of lunar simulants. The spectra of many of these simulants were reported earlier by Gaier, et al., ${ }^{3}$ but the results were confounded by a large variation in particles size.

The effects of particle size on the $\rho(\lambda)$ of lunar dust particles is illustrated in Fig. 2, which shows bidirectional reflectance spectra measured of a mare soil (12001) and a highlands soil (64801) returned by Apollo 12 and 16, respectively as reported by the Lunar Soil Characterization Consortium on their website. ${ }^{4}$ Note that the $\rho(\lambda)$ increases as the particle size decreases. The authors attributed this, at least in part, to their observation that the finer fraction contained more feldspar, which is lighter in color than the more mafic pyroxenes.

But the Gaier et al., study found that, with a single exception, all of the large grained simulants had higher $\alpha$ values than all of the small grained simulants. The composition of the simulants was nearly invariant between the larger and smaller components because the fine fractions were produced from grinding the coarser fractions. The present study was undertaken with the aim of eliminating particle size as a factor in the $\alpha$ and $\varepsilon$ determination by grinding and sieving the simulants to a common size range, $<20 \mu \mathrm{m}$. It was recognized that the grinding and sieving process has its own perils, such as the creation of more glassy phase and the possibility that softer minerals would be over-represented in the sieved fraction. Long grinding times were used to minimize the selective removal of the harder minerals, at the risk of increasing the glassy phase content. X-ray diffraction of the simulants before and after sieving was used to judge whether there was selected removal of major mineral components. The reflectance spectra in the $250 \mathrm{~nm}$ to $25 \mu \mathrm{m}$ range, the integrated solar $\alpha$, and the $300 \mathrm{~K} \varepsilon$ values are reported herein for a variety of ground and sieved lunar simulants.

\section{Methods and Materials}

\section{A. Spectroscopic Methods}

In order to determine the $\alpha$ of a simulant powder, the $\alpha(\lambda)$ over the solar spectrum must be determined. The easiest way to do that is to measure its $\rho(\lambda)$ and apply Eq. (2). Since on the lunar surface sunlight will impinge on the dust particles both directly and from many different reflected directions, the total $\rho(\lambda)$, including the specular
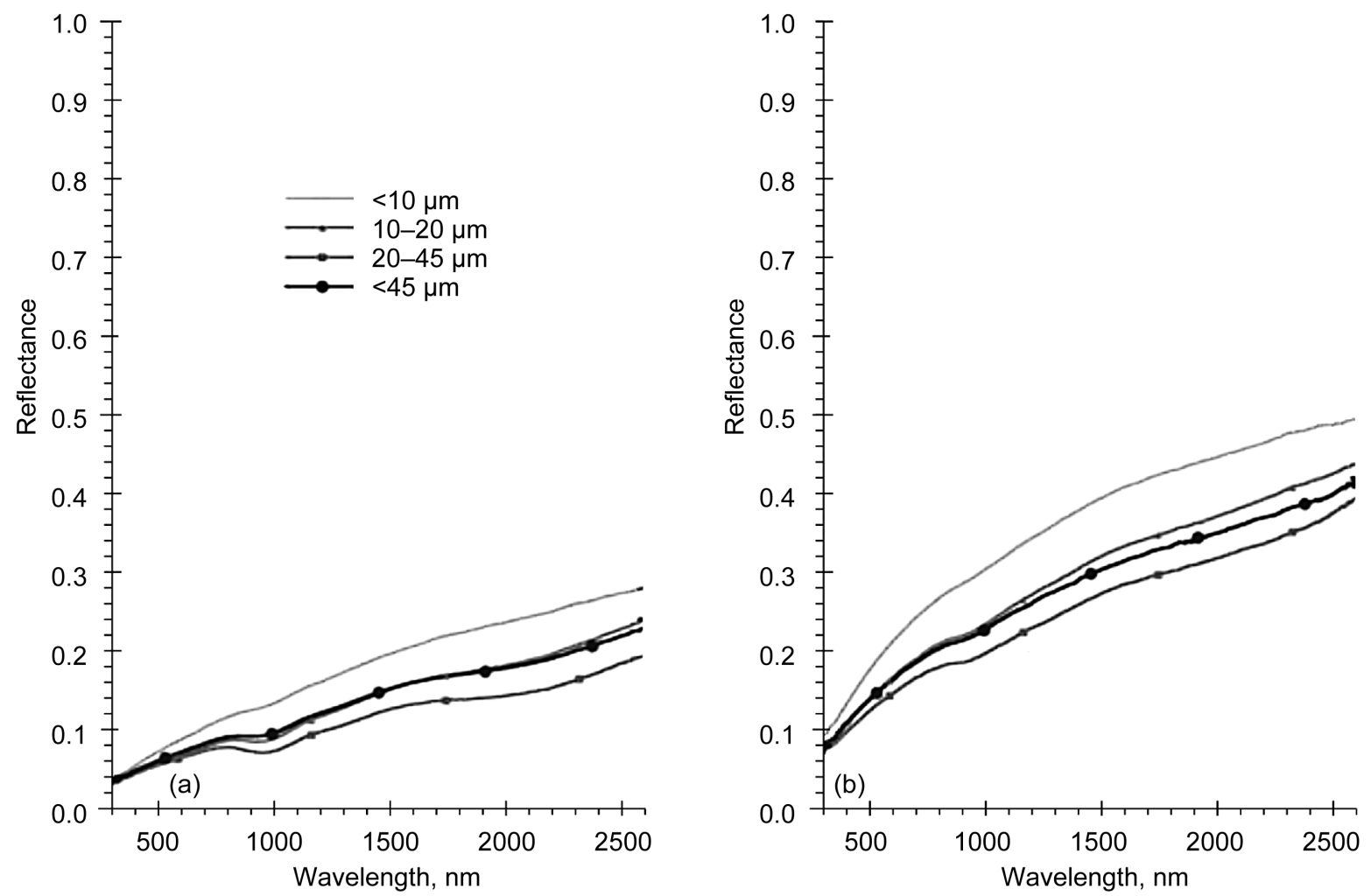

Figure 2. The bidirectional reflectance spectra (a) Apollo 12001 mare and (b) Apollo 64801 highland lunar soils. $^{2}$ 
and diffuse components is the quantity of interest. This is best accomplished using a spectrophotometer equipped with an integrating sphere. The reflectance spectra reported here were collected on a Cary 5000 spectrophotometer (Varian) equipped with a DRA 2500, $150 \mathrm{~mm}$ diameter integrating sphere over the wavelength range of 250 to $2500 \mathrm{~nm}$.

Great care must also be taken in the preparation of the sample. How tightly the powder is packed will make a difference in this wavelength range, with lose powders reflecting less. In this study a Varian powder attachment was used. A sample is trapped in a compression cell, one side of which is a quartz window. The cell is tightened to the point where the powder makes a smooth and uniform surface, and the grains do not move when the cell is tipped or rotated. The Varian powder attachment was filled with about 10 grams of sample which resulted in a sample about 4 $\mathrm{mm}$ thick, rendering all of the samples opaque.

Determining the scale factors to put the $\rho(\lambda)$ measurement on an absolute must be done thoughtfully as well. The 100 percent reflectance must be set with a powdered standard, which in turn must be calibrated against a NISTtraceable standard. Ideally, this calibration should be against a suite of NIST-traceable standards of varying $\rho(\lambda)$ to determine the response function of the detector. In this study a second, manufacture-provided powder attachment filled with powdered Spectralon ${ }^{\circledR}$, the same material that coated the inside of the integrating sphere, and a quartz window matched with the sample holder was used for the baseline. A set of eight NIST traceable diffuse reflectance standards (Labsphere) with $\rho$ ranging from 0.02 to 0.99 was measured through the quartz window as well. A correction factor was applied to the averaged $\rho$ for each sample to place it on an absolute scale.

Data were collected from 250 to $2500 \mathrm{~nm}$ in increments of $1 \mathrm{~nm}$, at a scan rate of $600 \mathrm{~nm} / \mathrm{min}$. A deuterium lamp was used to measure the 250 to $350 \mathrm{~nm}$ data, and a halogen lamp to measure the 350 to $2500 \mathrm{~nm}$ data. Immediately prior to running each sample, a spectrum of the Spectralon ${ }^{\mathbb{B}}$ was collected as a sample, to determine whether the baseline was still valid. In all instances the deviations in the baseline were less than 1 percent. Three spectra of each sample were collected consecutively. The sample holder was rotated between each spectrum so that the beam sampled a different area of the sample. If the three spectra were determined to be similar enough, the three spectra were averaged, point by point, and multiplied by the ratio of blank to baseline.

The integrated solar $\alpha$ of each sample was determined by calculating the convolution of the scaled $\rho(\lambda)$ of the simulant with the ASTM air mass zero solar spectrum E-490-00 and expressed as a fraction of the solar spectrum.

In order to determine the thermal $\varepsilon$, a similar procedure is required in the thermal infrared part of the spectrum. The $\varepsilon(\lambda)$ was determined from the $\alpha(\lambda)$ using Eq. (3), which in turn was determined from the $\rho(\lambda)$ using Eq. (2). Ideally the thermal infrared $\rho(\lambda)$ spectra should overlap with the solar $\rho(\lambda)$ spectrum (i.e., starting at less than $2.5 \mu \mathrm{m}$ ) and extend up to the wavelength that corresponds to the lowest temperature expected (i.e., $115 \mu \mathrm{m})$. But due to practical instrument considerations, it is difficult to measure infrared $\rho(\lambda)$ at wavelengths longer than about $25 \mu \mathrm{m}$.

Ambient laboratory air contains enough carbon dioxide and water to absorb the infrared in the light path, and distort the $\rho(\lambda)$ spectrum. So the integrating sphere was flooded with dry nitrogen gas to reduce absorption enough that the $\rho(\lambda)$ signal of the simulant could dominate. For this study reflectance spectra in the 2.5 to $25 \mu \mathrm{m}$ range were collected on a Magna-IR 760 (Nicolet) equipped with a Labsphere RSA-NI-550ID 76 mm Infragold ${ }^{\circledR}$ integrating $^{2}$ sphere using a deuterated triglycidyl sulfate detector. This is a Fourier transform instrument and 80 scans with a data point spacing of $7.714 \mathrm{~cm}^{-1}$ were collected for each sample. A gold background spectrum was measured prior to each scan. Powder samples of 4 to $6 \mathrm{~mm}$ thickness were place in a Fluoroware dish which was placed at the sample port at the bottom of the integrating sphere. No window was necessary in this configuration. None of the Fluoroware spectral peaks were visible in any of the spectra, indicating that the samples were optically opaque. During the course of the data collection two NIST traceable standards of similar reflectivity (SRS-10-010 and SRS-20-010) were measured as well.

There are subtle differences between the ways the data were collected on the two spectrophotometers. In the Cary, the sample was physically substituted for the reference on the surface of the integrating sphere. But in the Nicolet, the sample was measured by alternating a mirror between reference and sample positions. So in the case of the Nicolet, some small fraction of the integrating sphere when the reference was being measured was covered with the sample, whereas in the Cary it was not. This correction factor is dependent on the reflectance of the sample. Both instruments measured the samples at 1600 to $2500 \mathrm{~nm}$, so the average $\rho(\lambda)$ over this region was used to scale the Nicolet data to the Cary data. A plot of the Cary data as a function of the Nicolet data revealed that the $\rho(\lambda)$ varied linearly with an $\mathrm{R}^{2}$ of 0.97 . This correction factor was applied to all of the Nicolet data. The integrated thermal $\varepsilon$ was determined over the full temperature range expected on the lunar surface, 100 to $400 \mathrm{~K}$. This was calculated by convoluting the 2.5 to $25 \mu \mathrm{m}$ wavelength $\varepsilon(\lambda)$ data with blackbody curves over the temperature range in $5 \mathrm{~K}$ increments. ${ }^{5}$ The temperature dependence was small, so only the $\varepsilon$ at $300 \mathrm{~K}$ is reported here. 


\section{B. Lunar Simulants}

The basic spectra of six of the most common lunar simulants were measured, three mare type and three highlands type. The first of these was JSC-1A, a mare-type simulant that is a remake of the JSC- 1 simulant that has been the de facto NASA simulant since it was introduced in $1994 .{ }^{6}$ The second was a simulant developed by researchers at the University of Minnesota in 1988 approximating the bulk chemistry of Apollo 11 soil sample 10084, Minnesota Lunar Simulant \#1 (MLS-1). ${ }^{7}$ The third was developed in Japan in 1995 from the volcanic soils on Fujisan called FJS- $1 .{ }^{8}$ The fourth was a lunar highlands type simulant produced through a current joint effort of NASA and the U.S. Geological Survey, NU-LHT-2M. ${ }^{9}$ Two recently developed simulants from Canada were tested as well, the OB-1 and the Chenobi highlands simulants. ${ }^{10}$

In addition to those simulants, four simulant variants were measured to determine the general effects of adding minor constituents to the simulants. Since JSC-1A is a remake of JSC-1, their spectra should be identical. This was examined. MLS-1P is a variant of MLS-1 produced by dropping the MLS-1 through a $6000{ }^{\circ} \mathrm{C}$ plasma in an inflight sustained shockwave plasma reactor, $10 \mathrm{~m}$ tall. MLS-1 itself has essentially no glass content, but glass content from 10 to 30 percent was achieved with feed rates of 40 to $50 \mathrm{~kg} / \mathrm{hr}$. Although no agglutinate-like materials were produced, part of the simulant was melted into glassy spheroids, some as large a $2 \mathrm{~mm}$. ORBITEC, under a Phase I SBIR contract, used a proprietary process to create a mature lunar regolith simulant designated JSC $-1 \mathrm{~A}-5000-2 \mathrm{X}$ that contains a high proportion of agglutinate-like particles and glass spherules, both of which contain metallic iron globules (including nanophase $\mathrm{Fe}^{0}$ ). They also processed NU-LHT-2M in a similar manner to create a highlands simulant, designated NU-LHT-2M-700-1X containing simulated agglutinates with iron globules. ${ }^{11}$

Finally, the spectra of five minerals from which lunar simulants may be fabricated, anorthosite, norite, ilmenite, chromite, and harzburgite were measured as well. The hope is that models can be developed from these spectra that will enable the mimicking of various lunar dust spectra by the physical combination of these constituents.

\section{Processing the Lunar Simulants}

Particle size is known to affect the $\rho(\lambda)$ of powdered rock and mineral samples. This was dramatically shown in the case of lunar regolith in the data reported by the Lunar Soil Characterization Consortium, who measured the $\rho(\lambda)$ of sieved fractions of lunar samples returned during Apollo. ${ }^{4}$ They found that smaller particles were both more reflective and showed fewer spectral features. Since the simulants were acquired with a wide variety of particle sizes, they were processed to remove particle size as an important factor. Each of the samples was dry ground in a Micronizer (McCone) vibrating mill for $90 \mathrm{~min}$, and then sieved through a $25 \mu \mathrm{m}$ sieve (no 450). After both sets of spectral measurements were taken, $x$-ray diffraction analysis was performed on both the ground samples and the ground-and-sieved samples to make sure that the sieving was not systematically removing individual components from the samples.

X-ray diffraction (XRD) analysis was carried out on a Bruker D8 Advance diffractometer equipped with a sample spinner and a linear position-sensitive detector using $\mathrm{Cu} \mathrm{K}_{\alpha}$ radiation. The micronized materials were sampled before and after being sieved. A thin layer of each sample was dispersed with alcohol onto a silicon "zero background" holder and allowed to dry. Each sample was scanned over the range of 10 to $130^{\circ} 2 \theta$ with a step size of $0.02^{\circ}$ and a scan rate of $1 \mathrm{step} / \mathrm{s}$.

None of the samples showed differences in the major phases induced by sieving greater than 1 percent except for MLS-1P, which showed an increase of pyroxene over plagioclase by about 5 percent on sieving. This sample differed from the others in that the initial material contained glassy spheres up to $2 \mathrm{~mm}$ in diameter, much larger than the grain size. The mirconizing did not appear to significantly increase the size fraction of the glassy phase for any of the samples, as determined by XRD.

\section{Results and Discussion}

\section{A. Reflectivity Measurements}

The reflectance spectra of Chenobi, NU-LHT-2M, MLS-1, JSC-1AF, FJS-1, and OB-1 sieved to below $25 \mu \mathrm{m}$ particles are shown in Fig. 3. Large differences are noted among the six basic simulants in the $\rho(\lambda)$ in the 250 to $2500 \mathrm{~nm}$ range, where the solar spectrum is the most intense. Even before a quantitative analysis, it is apparent from these spectra that there will be substantial variations in the solar $\alpha$ among the simulants. As expected, two of the highlands type simulants, Chenobi and NU-LHT-2M are more reflective than the mare types. OB-1, despite the fact that it was intended to be a highlands type simulant, proved to be among the darkest of the simulants.

It is clear that there was little difference in the $\rho(\lambda)$ among the simulants at wavelengths greater than about $8 \mu \mathrm{m}$, except for the case of MLS-1. Further, the infrared $\rho(\lambda)$ for those simulants is about 0.10 for wavelengths longer than $8 \mu \mathrm{m}$. Using the Raleigh-Jeans law this corresponds to temperatures of $360 \mathrm{~K}$ and lower, so for the 
temperatures at which the thermal control surfaces are expected to operate under lunar conditions, the $\varepsilon$ 's are all about the same. This implies that the thermal emission properties all over the lunar surface are likely to be similar, and in the range of 0.9 .

It can be seen in Fig. 3(b) that MLS-1 is much more reflecting than the other simulants in the 10 to $25 \mu \mathrm{m}$ wavelength range. This is thought to be because MLS-1 contained almost no glass or agglutinates. Glasses are known to dramatically increase absorption over crystalline materials in this portion of the spectrum. In addition, the other simulants all had high glass content, as does the lunar soil. So MLS-1 would probably not be a good simulant to use if the $\varepsilon$ properties are important to the test. But MLS-1P is a variant of this material that has been exposed to a high temperature plasma, in which part of the MLS-1 is melted, and quickly cooled to increase its glass content. Fig. 4 shows the darkening that this produces over the entire spectral range.

The participants at the Lunar Regolith Simulant Materials Workshop convened by NASA in 2005 agreed that engineering large quantities of lunar simulants would be required to support NASA's Exploration program. Since quantities of the mare simulant JSC-1 were largely depleted, it was decided to recreate that simulant, with the
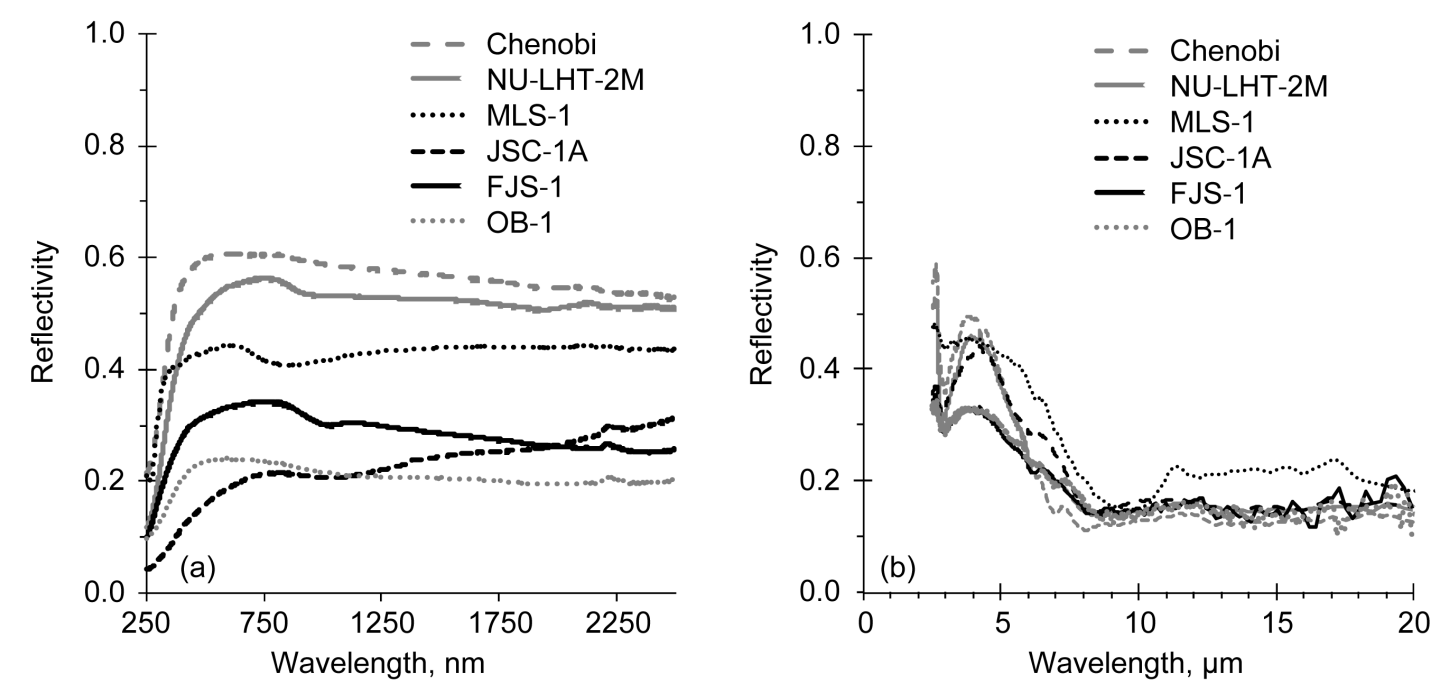

Figure 3. Reflectance spectra of the six baseline simulants measured in this study in the solar absorptance region (a), and for the emittance regions (b).
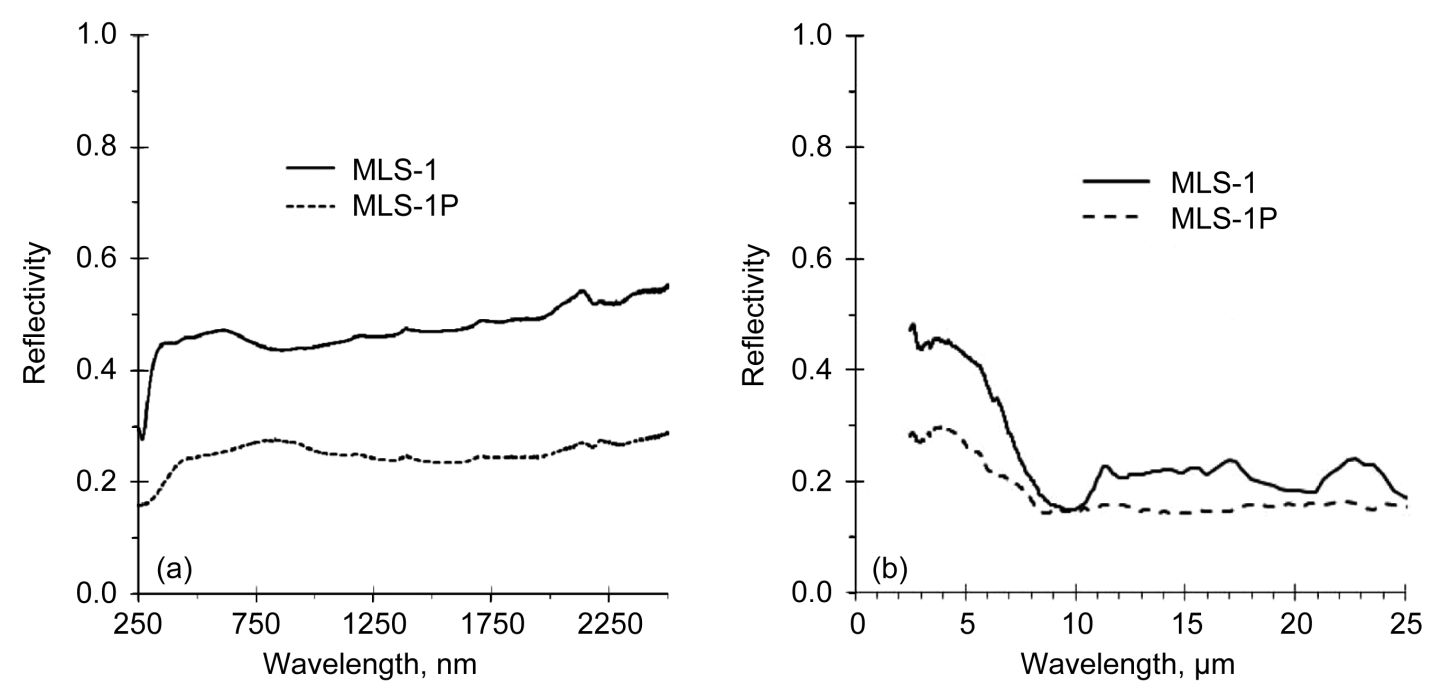

Figure 4. Reflectance spectra of the MLS-1 and MLS-1P in the solar absorptance region (a), and for the absorptance and emittance regions (b). 
designation JSC-1A. Although the goal was an exact reproduction of JSC-1, it can be seen in Fig. 5 that the reflectance spectrum, particularly in the 250 to $2500 \mathrm{~nm}$ range, differs appreciably. This illustrates an important lesson about the variability of natural materials. Even if the source material is taken from the same region and processed using the same techniques, there will be differences. So too will it be on the Moon. It will be more useful to consider the range of material properties the lunar regolith will have than to try to duplicate one particular sample exactly.

Unlike the lunar simulants in Fig. 3(a), the $\rho(\lambda)$ of lunar soils increases almost monotonically over the range from 300 to $2500 \mathrm{~nm}$ (Fig. 2). This is often attributed to agglutinitic glass, and the nanophase iron it contains. So the addition of synthetic agglutinates, which also contain small metallic iron particles would be expected also to increase the $\rho(\lambda)$ over the base simulant in a similar way. Figure 6 shows $\rho(\lambda)$ for JSC -1 A and its variant JSC -1 A- $-5000-2 X$, which had synthetic agglutinate particles added to it. Although there was an appreciable increase in the $\rho(\lambda)$ for this variant in the 250 to $2500 \mathrm{~nm}$ range, there was little effect on the reflectivity at wavelengths greater than $8 \mu \mathrm{m}$. Figure 7 shows $\rho(\lambda)$ for NU-LHT and its variant NU-LHT-2M-700-1X which has agglutinates added using the same method as JSC-1A5000-2X. Here, there was even a smaller effect of the synthetic agglutinates, if any at all, on the reflectance spectrum.
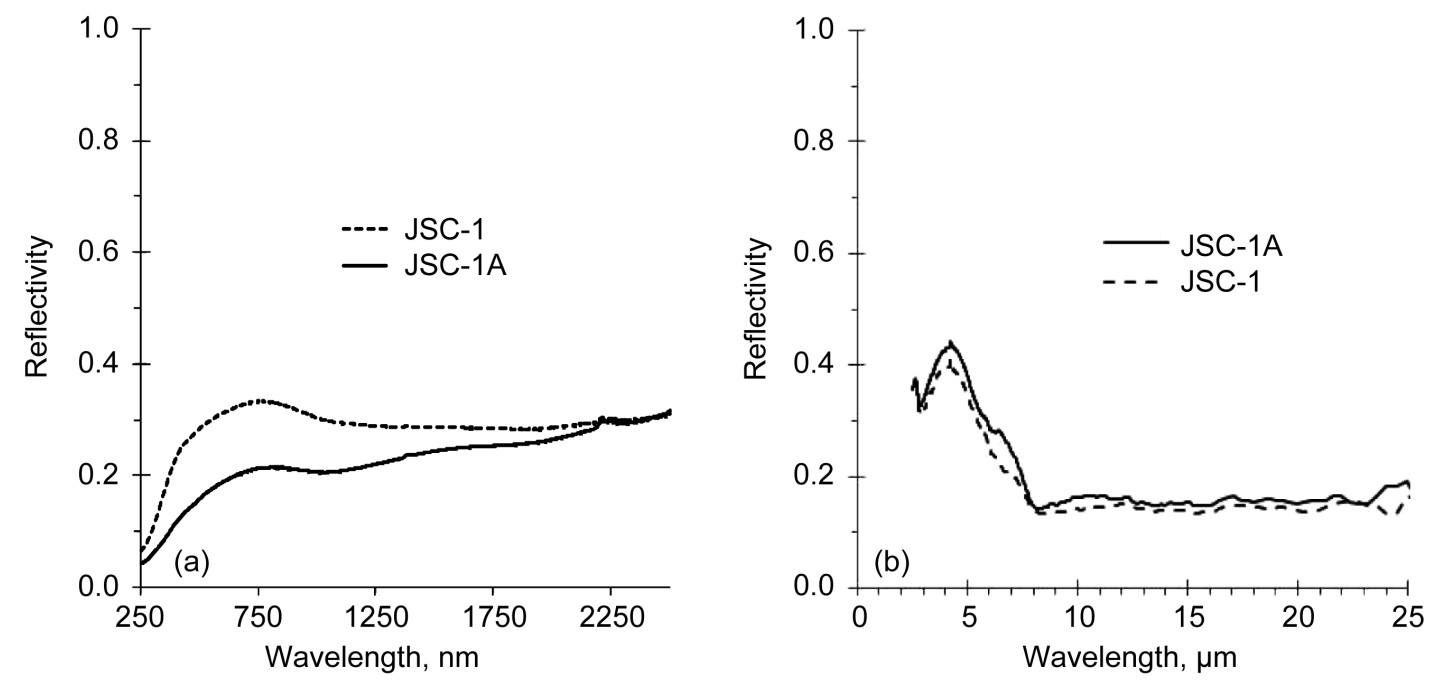

Figure 5. Reflectance spectra of the JSC-1 and JSC-1A in the solar absorptance region (a), and for the thermal emittance region (b).
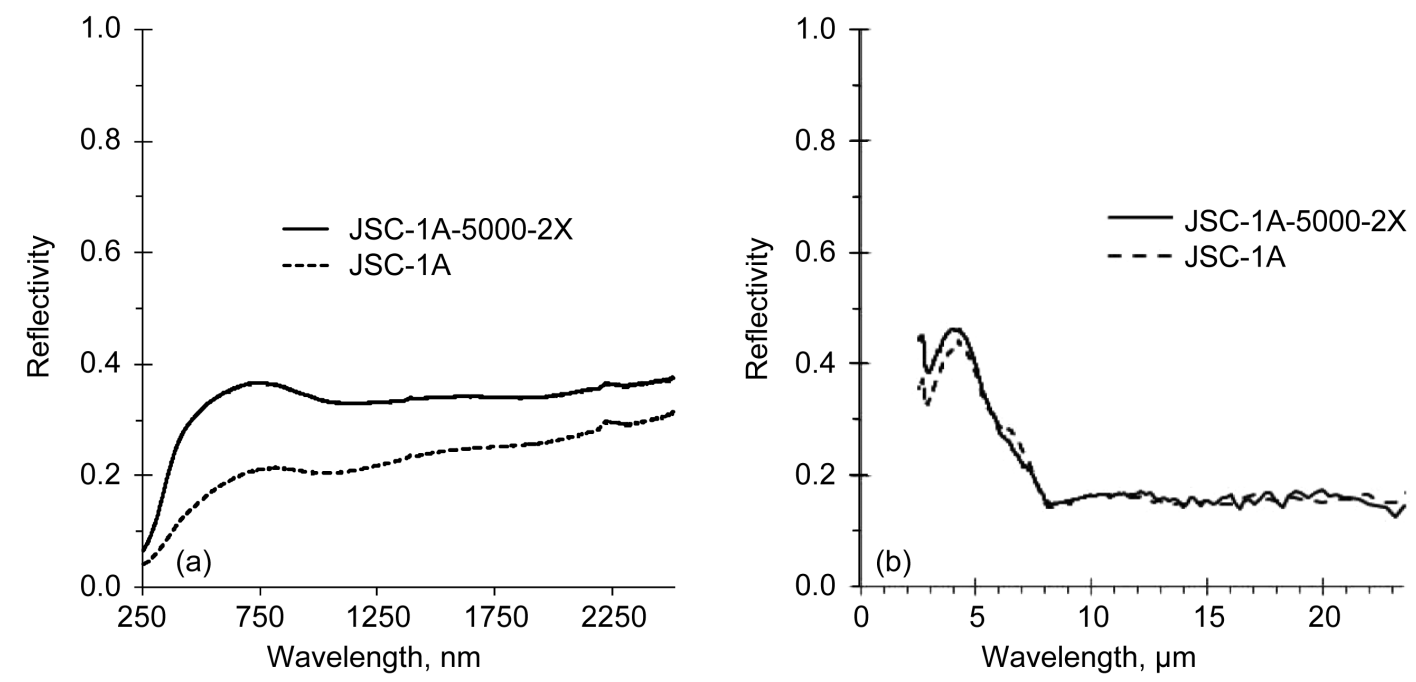

Figure 6. Reflectance spectra of the JSC-1A and the synthetic agglutinate variant JSC-1A-5000-2X in the solar absorptance region (a), and for the thermal emittance region (b). 

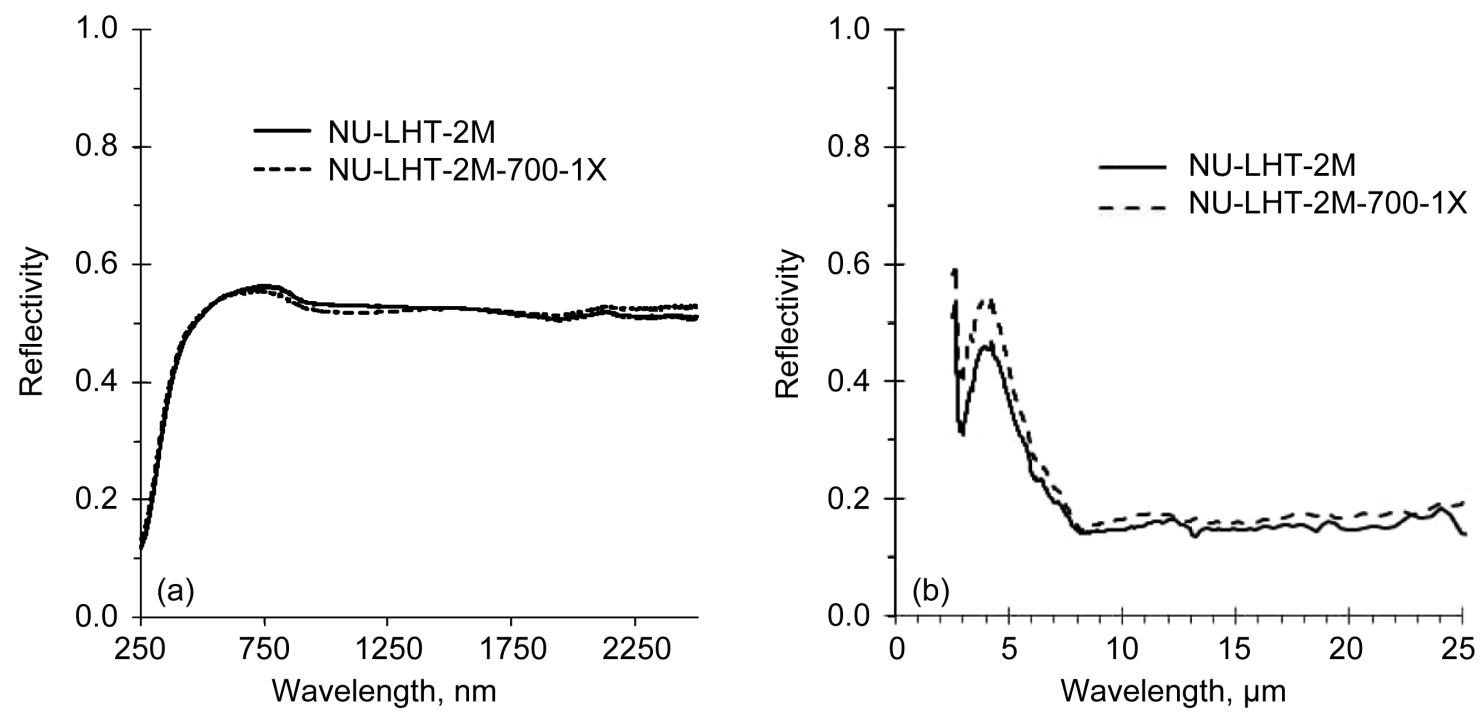

Figure 7. Reflectance spectra of the NU-LHT-2M and the synthetic agglutinate variant NU-LHT-2M-700-1X in the solar absorptance region (a), and for the thermal emittance region (b).

There are at least two possible explanations why the spectra of the simulants did not change as expected when the agglutinates were added. The first is that the synthetic agglutinates do not reproduce the key characteristics of the lunar agglutinates in sufficient fidelity. The most difficult part of the lunar agglutinate to reproduce is the nanophase iron which is found primarily in the amorphous "rind" around each grain, but also to a lesser extent on the grain surface. Even though nanophase iron has been observed in the transmission electron microscope, the particle size and distribution of the nanophase may be critical in some manner that is not as yet understood. The second explanation is that the effect in lunar materials may be caused by factors in addition to agglutinate and nanophase iron that are not as yet understood.

Like most lunar soils, the lunar simulants fall into two broad categories, the darker mare simulants and the lighter highlands simulants. In order to determine whether the effects on thermal control systems vary linearly with the darkness of the soil, a simulant of intermediate $\alpha$ was desired. To accomplish this a 1:1 mixture of the light highlands simulant NU-LHT-1D and the dark mare simulant JSC-1AF was prepared. This also provided an opportunity to see how additive the $\alpha$ and $\varepsilon$ of these multicomponent mixtures are. The results are shown in Fig. 8, which shows the mixed simulant spectrum along with those of its constituents. Also shown is a point by point average of the two. It can be seen that the point-by-point average lies nearly on top of the 1:1 mixture, showing that the $\rho(\lambda)$ is additive.

Many lunar simulants are not simply a similar mineral composition that is dug out of the ground, but a mixture of minerals which may come from a variety of sources and geographic locations. This is entirely appropriate since the soils returned during the Apollo missions were composed of a variety of granular materials including single crystal grains, volcanic and impact glasses, multi-mineral grains, and agglutinates. Figure 9 shows the spectra of four components that have been found in lunar materials and could be used to develop lunar simulants. Anorthosite and norite are plagioclase feldspars of the type that make up the bulk of the lunar regolith. Harzbergite is made up of pyoxenes and olivine, the principal minerals that darken the regolith. Ilmenite and chromite are representative of very dark minor constituents. Since the thermal optical spectra are additive, the spectra of combinations of lunar separates can be predicted. So if a target spectrum of a region of the lunar surface can be determined, in principal that spectrum can be duplicated by combinations of mineral and glassy separates. However, it is noted that none of these components have the characteristic rising $\rho(\lambda)$ with $\lambda$ which is characteristic of lunar soils (see Fig. 2). It is thought that perhaps it is the agglutinates and associated nanophase iron that give rise to this type of spectrum, but to date no material with rising $\rho(\lambda)$ has been duplicated in the laboratory. 


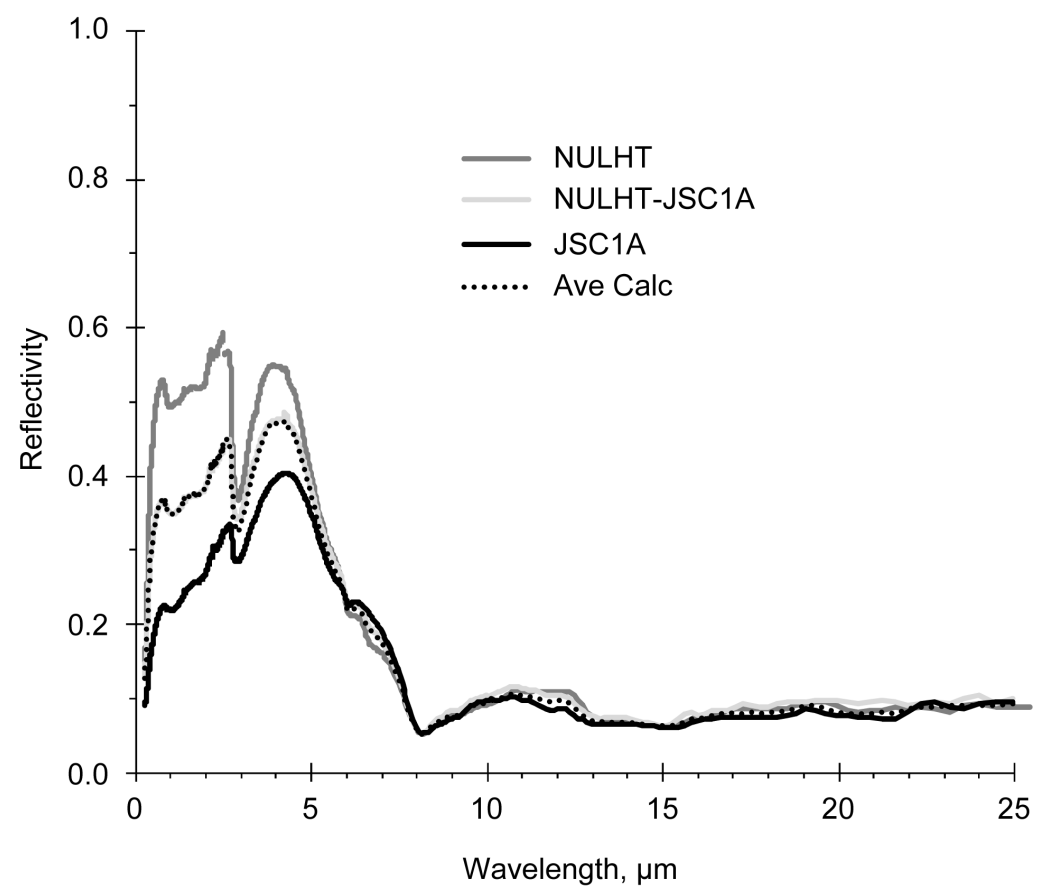

Figure 8. The reflectance spectra of JSC-1AF, NU-LHT-1D and a 1:1 mixture of the two in the solar absorptance region (a), and for the absorptance and emittance regions (b). The dotted line shows the point by point average of JSC-1AF and NU-LHT-1D in the solar absorptance region (a), and for the absorptance and emittance regions (b).
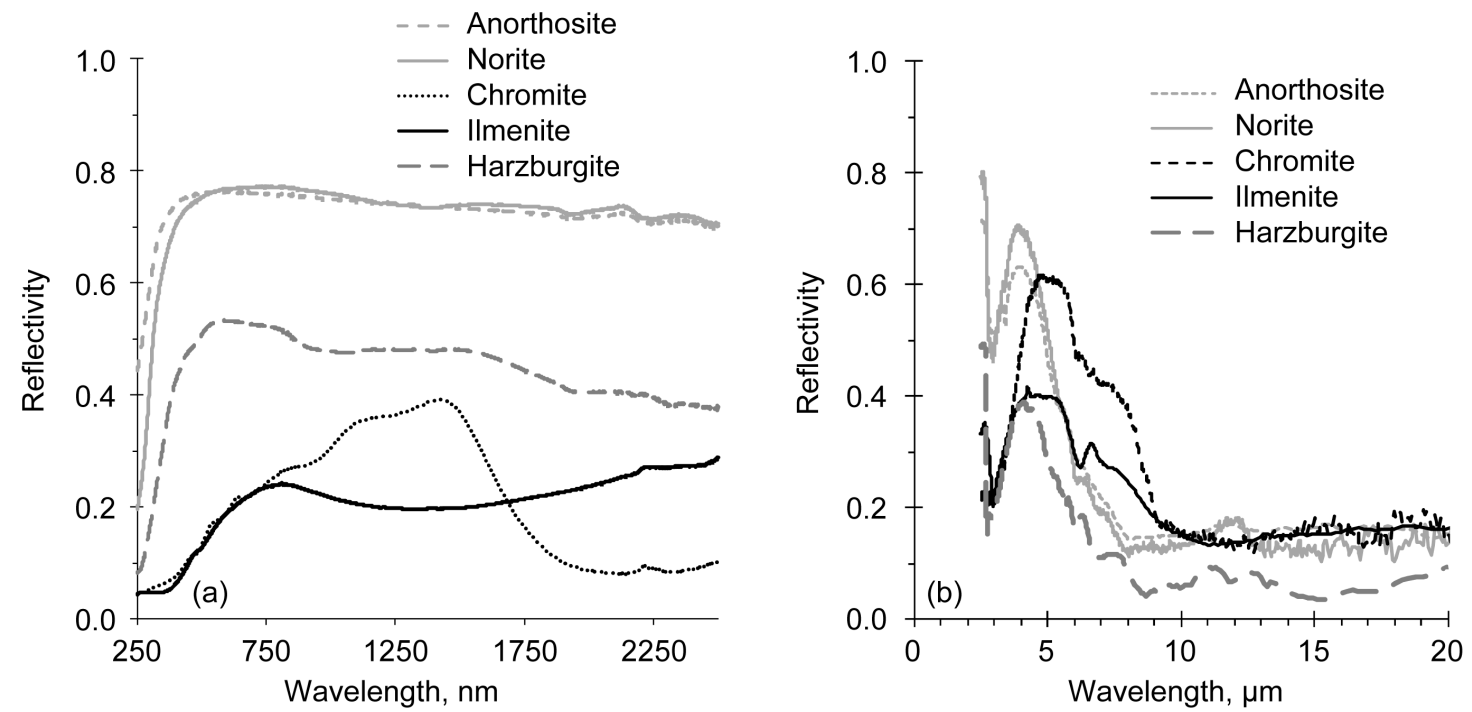

Figure 9. Reflectance spectra of the constituent minerals anorthosite, norite, chromite, ilmenite, and harzburgite in the solar absorptance region (a), and in the thermal emittance region (b).

\section{B. Thermal Optical Property Calculations}

Since the goal for this series of tests is to characterize the thermal properties of the simulant dust, the $\rho(\lambda)$ for each must be used to calculate the $\alpha$ and $\varepsilon$. The 250 to $2500 \mathrm{~nm}$ data were used to calculate the $\alpha$, and the 2.5 to $25 \mu \mathrm{m}$ data were used to calculate the $\varepsilon$. The $\alpha$ and $\varepsilon_{300}(\varepsilon$ at $300 \mathrm{~K})$ for each of the simulants are listed in Table 2 .

The $\alpha$ ranged from 0.39 to 0.75 for the simulants. Albedo measurements suggest a range of $\alpha$ for the Moon of 0.50 to 0.93 . As discussed above, the albedo measurements tend to indicate $\alpha$ values that are somewhat higher than expected from measuring the intrinsic $\alpha$ of the dust. In addition, these simulants would compare to the smallest fraction of the regolith, and for that reason would tend to be high. An appreciation for the impact of the particle size 
effect for these materials can be gained by comparing these $\alpha$ values to those of the samples in their as-received sizes, as reported by Gaier, et al. ${ }^{3}$ which are also shown in the table for comparison. The finest fraction of the mare soil 12001 has an $\alpha \approx 0.89$, and the coarsest fraction about 0.91 , within the ranges of the coarser mare simulants tested here, but darker than the simulant fine fraction. The $\alpha$ of the highland soil 64801 varies from about 0.74 for the finest fraction to about 0.80 for the coarsest fraction, considerably darker than the range of the mare simulants tested. It is expected that the NU-LHT-2M would be lighter than typical highlands samples because the particles do not have any of the characteristics of space weathering, and thus represent fresh, rather than matured soils. The NULHT-2M-700-1X has had synthetic agglutinate glass and iron particles added, and thus is somewhat darker, at least for the larger particle sizes. However, the micronizing and sieving appears to have resulted in a simulant that is no darker in the fine fraction. However, some of the mare simulants, most notably JSC-1A, appear to mimic the optical properties of the highlands soil quite well. So when simulating the effects of dust and soil on thermal control surfaces, matching the simulants to the optical properties is a better strategy than merely using a highlands simulant for the highlands region, or a mare simulant for a mare region.

Table 2. Integrated solar absorptance $(\alpha)$ calculated from 250 to $2500 \mathrm{~nm}$ total reflectivity and integrated thermal emittance $(\varepsilon)$ at $300 \mathrm{~K}$ calculated from 2.5 to $25 \mu \mathrm{m}$ total reflectivity. Values for $<20 \mu \mathrm{m}$ samples are from this study, and those for $>20 \mu \mathrm{m}$ samples from the work of Gaier, et al. ${ }^{3}$ Values from lunar soil samples ${ }^{*}$ ) calculated 300 to $2500 \mathrm{~nm}$ using bidirectional reflectance spectra from $\mathrm{LSCC}^{4}$.

\begin{tabular}{lcccc}
\hline \hline Simulant & $<\mathbf{2 0} \boldsymbol{\mu} \mathbf{m} \boldsymbol{\alpha}$ & $\mathbf{2 0} \boldsymbol{\mu \mathbf { m }} \boldsymbol{\alpha}$ & $<\mathbf{2 0} \boldsymbol{\mu \mathbf { m }} \boldsymbol{\varepsilon}$ & $>\mathbf{2 0} \boldsymbol{\mu \mathbf { m }} \boldsymbol{\varepsilon}$ \\
\hline Chenobi & 0.39 & --- & 0.86 & --- \\
NU-LHT-2M & 0.45 & 0.56 & 0.78 & 0.88 \\
NU-LHT-2M-700-1X & 0.46 & 0.67 & 0.82 & 0.90 \\
MLS-1 & 0.54 & 0.82 & 0.73 & 0.70 \\
MLS-1P & 0.75 & 0.85 & 0.84 & 0.90 \\
JSC-1 & 0.64 & --- & 0.84 & --- \\
JSC-1A & 0.75 & 0.82 & 0.83 & 0.90 \\
JSC-1A-5000-2X & 0.66 & 0.92 & 0.83 & 0.91 \\
FJS-1 & 0.65 & 0.81 & 0.84 & 0.90 \\
OB-1 & 0.73 & --- & 0.92 & -- \\
Mare soil 12001* & 0.89 & 0.91 & & \\
Highlands 64801* & 0.74 & 0.80 & & \\
\hline \hline
\end{tabular}

The $\varepsilon$ at $300 \mathrm{~K}$ for each of the simulants is given in Table 2. If MLS- 1 is excluded, the $\varepsilon$ 's of the fine fraction of the lunar simulants ranged from 0.78 to 0.92 with an estimated error of \pm 0.005 . This is about 0.1 lower than was found with the larger particles sizes. The higher value for the $\rho(\lambda)$ in MLS-1 is probably due to a lower level of glassy material, which is known to decrease the $\rho(\lambda)$ in the infrared. The fact that MLS- 1 with smaller particle sizes actually had a higher $\varepsilon$ may be an indication that a glassy phase is generated upon milling. Since the lunar dust contains a very high fraction of impact glasses, the $\varepsilon$ of the lunar dust would be expected to be high, and has been found to be about 0.95 . The $\varepsilon$ of the dust will also be somewhat higher than that of the thermal control surfaces, and so the blocking of emitted heat will probably not play a major role in thermal performance degradation. Nearly all of the dust degradation of thermal control surfaces will be due to increased $\alpha$, that is, the dust will keep the solar incident light from reflecting off of the thermal control surface. This is consistent with what was observed on the Apollo LRV radiators when they were dust covered.

\section{Conclusions}

A comparative study of the reflectance spectra of lunar simulants is presented. All of the simulants except one had a wavelength-dependant reflectivity $(\rho(\lambda))$ near 0.10 over the wavelength range of 8 to $25 \mu \mathrm{m}$, so they are highly emitting at room temperature and lower. The $300 \mathrm{~K}$ emittance $(\varepsilon)$ of all the lunar simulants except one ranged from 0.88 to 0.91 . There was considerably more variation in the lunar simulant reflectance in the solar spectral range $(250$ to $2500 \mathrm{~nm})$ than in the thermal infrared. As expected, the lunar highlands simulants were more reflective in this wavelength range than the lunar mare simulants. The integrated solar absorptances $(\alpha)$ of the simulants ranged from 0.39 to 0.75 . This is lower than the values reported earlier for larger particles of the same simulants, and for 
representative mare and highlands lunar soils. It is recommended that $\alpha$ and $\varepsilon$ values be the criteria for choosing a simulant for assessing the effects of dust on thermal control surfaces, rather than whether a simulant has been formulated as a highlands or a mare simulant.

\section{Acknowledgements}

The authors gladly acknowledges the contributions of K. Street, S.K.R. Miller and D.A. Jaworske of the NASA Glenn Research Center (GRC), D. Rickman of the NASA Marshall Spaceflight Center, and D. Stoesser of the U.S. Geological Survey for their many helpful discussions about the properties of the lunar regolith and lunar simulants. S. Wilson of the U.S. Geological Survey and R.J. Gustafson of Orbital Technologies kindly provided simulant samples. Assistance with the x-ray diffraction data collection and analysis was provided by R. Rogers and M. Cox of GRC. Programmatic and financial support from the NASA Exploration Technology Development Program came through the Dust Mitigation Project (M.J. Hyatt at GRC) and the Advanced Thermal Control Project (R.A. Stephan, NASA Johnson Space Center.)

\section{References}

1“Apollo 16 Preliminary Science Report,” NASA SP-131. (Bret R., England A. W., Calkins J. E., et al.). Washington D.C.: NASA; 1972.

${ }^{2} 2000$ ASTM Standard Extraterrestrial Spectrum Reference E-490-00. 2000. Available at http://rredc.nrel.gov/solar/spectra/AM0.

${ }^{3}$ James R. Gaier, Kenneth W. Street, and Robert J. Gustafson, "Measurement of the Solar Absorptance and Thermal Emittance of Lunar Simulants," International Conference on Environmental Systems 2010, AIAA-2010-6025. Also NASA/TM-2010-216788.

${ }^{4}$ Lunar Soil Characterization Consortium. Bidirectional reflectance spectra for lunar soils. Available at http://www.planetary.brown.edu/relabdocs/LSCCsoil.html, accessed April 2010.

${ }^{5}$ Jaworske D, Skowronski T., "Portable Infrared Reflectometer for Evaluating Emittance," Space Technology and Applications Forum, Ed., M.S. El-Genk, American Institute of Physics 2000:CP-504.

${ }^{6}$ McKay D., et al., "JSC-1: A New Lunar Soil Simulant," Engineering, Construction, and Operations in Space IV, American Society of Civil Engineers; 1994: 857-866.

${ }^{7}$ Weiblen P, Gordon K., "Characteristics of a Simulant for Lunar Surface Materials," Second Conference on Lunar Bases and Space Activities in the $21^{\text {st }}$ Century, Lunar and Planetary Institute; 1988:652.

${ }^{8}$ Kanamori H, et al., "Properties of Lunar Soil Simulant Manufactured in Japan," Proceeding of the $6^{\text {th }}$ International Conference and Exposition on Engineering, Construction, and Operations in Space. Albuquerque: ASCE; 1998.

${ }^{9}$ Stoesser D, et al., "Development of Lunar Highlands Type Simulants, NU-LHT-1M. -2M," Geochemica et Cosmochemica Acta. 72(12):A902.

${ }^{10}$ Battler M. M., Spray J. G., "The Shawmere Anorthosite and OB1 as Lunar Highlands Regolith Simulants," Planetary and Space Science. 2009: 57(15-15):2128-2131.

${ }^{11}$ Gustafson R, White B. "Development of a Lunar Dust Simulant," International Conference on Environmental Systems Proceedings. Society of Automotive Engineers; 2009:09ICES-0125. 